\title{
La Commission des principes s'exprime sur la question du «rationnement» dans le système des soins en Suisse
}

\author{
Commission fédérale \\ des principes de l'assurance- \\ maladie
}

En 2001, la Commission fédérale des principes de l'assurance-maladie (commission des principes) a mandaté deux études [1, 2] qui devaient permettre de savoir si les prestations médicales en Suisse subissent un rationnement implicite, et si oui, dans quelle proportion et sous quelle forme.

Lors de sa séance du 17 mars 2006, elle a discuté de ces deux documents et de la question du «rationnement» des soins puis, fin mai 2006, a définitivement pris position par circulaire. Les avis en question ont été rédigés par un groupe de travail composé de membres et de suppléants de la commission des principes [3].

\section{Prise de position sur les deux études concernant le rationnement implicite} Le rapport «Effects of Rationing of Nursing Care in Switzerland on Patients' and Nurses' Outcomes» de l'Institut pour la science des soins infirmiers de l'Université de Bâle (abrégé RICH dans la suite du texte) et l'étude «Is there evidence of implicit rationing in the Swiss health care system?», rédigée par B. Santos-Eggimann de l'Institut universitaire de médecine sociale et préventive de Lausanne (abrégée RIS - rationnement implicite en Suisse - dans la suite du texte), tentent de dépister un rationnement implicite en s'appuyant sur différentes approches et d'éventuels indices.

L'étude RICH se demande si, par manque de ressources chez l'équipe soignante, des soins essentiels n'ont pas été prodigués et si un lien peut être établi avec les indicateurs qualitatifs des soins et des prestations médicales. Elle examine en outre les conditions de travail et compare les résultats aux données internationales. Ces résultats indiquent clairement que la qualité des soins et des prestations médicales dans les hôpitaux (erreurs dans la remise de médicaments, chutes, infections nosocomiales, incidents critiques, ulcères de décubitus, mortalité, satisfaction des patients) dépend des ressources médicales disponibles et parfois aussi des conditions de travail évaluées par le personnel lui-même. Les hôpi- taux présentent des différences considérables non seulement du point de vue de la satisfaction du personnel soignant, mais aussi de la fréquence des erreurs évitables. En ce qui concerne la qualité des prestations et des conditions de travail, la Suisse se trouve en position relativement bonne par rapport aux données internationales.

L'étude RIS tente de déterminer, à partir des différents acteurs du système de santé (médecins et administrateurs d'hôpitaux) et des données de l'Enquête suisse sur la santé, à quel point les restrictions des possibilités de traitement nécessaire sont ressenties, notamment par rapport à l'accès aux prestations nécessaires. L'étude a relevé de fortes disparités cantonales et régionales pour l'accès à ces dernières, et cela particulièrement pour les services psychiatriques. Des groupes à risque, pour qui l'accès aux prestations médicales nécessaires semble compromis, ont été définis. Il s'agit des personnes âgées, des personnes mentalement handicapées, des personnes atteintes d'une maladie mentale et des personnes socialement défavorisées.

Pour le groupe de travail, il en est ressorti que:

1. Bien que la couverture médicale en Suisse soit de haut niveau, il existe de fortes disparités entre les cantons et entre les régions pour l'accès aux structures de soins (RIS).

2. Différentes structures du personnel dans les hôpitaux peuvent conduire à une perte de qualité (RICH).

3. Il faut accorder une attention toute particulière à certains groupes à risque comme les personnes atteintes d'une maladie mentale, les personnes âgées et les personnes mentalement handicapées (RIS).

4. Les études mettent notamment en évidence un besoin accru de surveillance de la qualité et de la sécurité des patients afin de réduire les différences (RICH et RIS).

5. Le recours plus systématique aux indicateurs de qualité peut aider à utiliser plus efficacement les outils du système de santé et à diminuer ainsi les inégalités. 


\section{Prise de position sur le rationnement dans le système de soins en général}

\section{Préambule}

Tout système de soins est réglementé afin de garantir la sécurité des patients et la qualité des prestations. De même, le financement des soins impose des choix visant à déterminer les prestations prises en charge par les assurances sociales ou privées ou par l'Etat. C'est ainsi que tout système de soins comprend des mesures de sélection des prestations, qui garantissent aux personnes un accès équitable à des soins de qualité répondant à ses besoins de santé. Le droit d'accès aux soins est donc soumis à restriction pour des raisons de police sanitaire, de qualité et de ressources disponibles.

\section{Principes}

1. On peut définir le rationnement comme un processus de choix, fondé sur des priorités parmi des services, des pratiques et des prestations efficaces et utiles, demandées ou considérées comme nécessaires par les patients ou les consommateurs d'une part, et par les fournisseurs de soins d'autre part. Un tel processus influence la prescription et la consommation de soins et donc l'accès au système de soins.

2. D'habitude, un processus de rationnement est mis en ouvre explicitement au sein du système de soins lorsque la demande, primaire ou induite, dépasse l'offre ou les ressources allouées, respectivement lorsque les coûts dépassent les ressources allouées. L'existence d'un processus de rationnement dépend ainsi autant de la demande et de l'offre, que de l'allocation des ressources.

3. Un processus de rationnement est implicite lorsque les priorités n'ont pas été définies ou décidées explicitement mais laissées au pouvoir discrétionnaire d'un prescripteur de soins (généralement un médecin ou un autre soignant) qui, grâce à l'asymétrie de l'information, peut décider seul ou, à tout le moins, influencer de manière décisive le traitement finalement appliqué. Le rationnement implicite peut aussi être induit par un manque de transparence dans l'allocation des ressources.

4. L'identification et la mesure de situations de rationnement implicite supposent, pour chaque situation de maladie ou de soins correctement diagnostiquée, l'existence de normes de bonne pratique (indications et prescriptions fondées sur un large consensus scientifique) telles qu'on devrait les appliquer hors de toute situation de rationnement.
5. La certitude de l'existence d'un rationnement implicite pour un patient donné suppose donc au minimum une évaluation de l'état de santé de ce patient et la mise en évidence d'un écart entre le traitement appliqué et un traitement considéré comme la règle de l'art. 6. En général, l'activité sanitaire est ou peut être caractérisée par la complexité, l'incertitude, une qualité difficilement mesurable, l'asymétrie de l'information, voire les conflits d'intérêts, l'autoritarisme et l'opacité des décisions et, du côté des consommateurs, par des attentes irrationnelles et magiques souvent soutenues par des états d'anxiété et d'angoisse et par des besoins de justice et de légitimité.

7. Cette situation engendre une grande variabilité des pratiques, des prescriptions et des consommations, variabilité étayée par une abondante littérature scientifique aussi bien au niveau suisse qu'international.

8. Ces études montrent des variabilités de la prescription (ou de la consommation) atteignant un facteur de 1 à 4 (+300\%), ce qui est une preuve indirecte du manque de consensus sur les indications et les «règles de bonne pratique» ou leur application parmi les professionnels.

\section{Conclusions}

9. Tirer de là, sur la base de la simple variabilité, des conclusions sur l'existence de situations de rationnement implicite serait une opération arbitraire si l'on ne connaît pas le taux «correct» de prescription ou de consommation d'une certaine prestation, au niveau individuel et/ou dans une population donnée.

10. En effet, en particulier dans un pays comme la Suisse qui assure, notamment par le biais de la LAMal, une très bonne égalité d'accès aux soins à toute sa population, on n'a pas d'informations fiables qui puissent nous faire dire que la population d'un canton avec un faible taux d'interventions chirurgicales soit à risque de rationnement implicite. En effet, par manque d'information sur le processus décisionnel, on pourrait aussi spéculer que dans les cantons qui connaissent des taux plus élevés d'interventions, on est en présence d'une surconsommation ou d'une offre trop importante de soins.

11. De ce fait les variabilités, notamment géographiques, constatées en Suisse dans l'accès à différentes prestations médico-sanitaire mettent en évidence des problèmes liés à la qualité et à l'adéquation des prestations et des indications, mais sans suffire pour don- 
ner des réponses quant à l'existence d'un rationnement implicite.

12. D'autres indicateurs liés à la gestion et aux objectifs d'un système de soins devraient être retenus pour donner une première réponse à la question de l'existence ou non d'un rationnement implicite. Ces indicateurs, pour la plupart de type socio-économiques, devraient notamment évaluer les obstacles et les entraves à un accès équitable et en temps utile aux prestations et aux services pour la population en général, et ses groupes les plus fragiles et les moins favorisés en particulier [4].

13. Afin de mieux appréhender le phénomène du rationnement implicite, il est nécessaire de:

a. définir des indicateurs susceptibles de faire apparaître une inégalité dans l'accès aux soins (listes d'attente en fonction du type d'assurance, du service, de la région, du revenu, de la franchise, des paiements informels, etc.);

b. procéder à un monitoring, avec bench marking, de ces indicateurs.

14. Parallèlement, il est nécessaire de:

a. définir les services prioritaires et «sensibles» en fonction de groupes et/ou sousgroupes de population vulnérables;

b. procéder à une collecte et une analyse systématiques des données selon les services prioritaires et les groupes et sous-groupes de population prédéfinis, ainsi qu'en fonction des indicateurs choisis.

15. Afin de limiter l'impact potentiellement négatif sur la santé de la population et des patients d'un éventuel rationnement implicite, il faudrait également:

a. donner sa cohérence au système de santé; b. définir les orientations du système de soins en fonction des besoins de santé de la population et non prioritairement en fonction des coûts des prestations;

c. augmenter la recherche dans l'évaluation des services et des politiques de santé dans un souci de transparence et d'égalité;

d. compte tenu des besoins de santé publique, étudier la possibilité de décider d'un remboursement temporaire de prestations, sous condition d'une évaluation visant à déterminer si elles répondent aux critères d'efficacité, d'adéquation et d'économicité.

Berne, Juin 2006

\section{Références}

1 Schubert M, Schaffert-Witvliet B, Glass T, De Geest S. Effects of Rationing in Nursing Care in Switzerland on Patients' and Nurses' Outcomes. Basel: Institute of Nursing Science, University of Basel 2004. (Unpublished report).

2 Santos-Eggimann B. Is There Evidence of Implicit Rationing in the Swiss Health Care System? Lausanne: Institute of Social and Preventive Medicine, University of Lausanne 2005. (Unpublished report).

3 Membres du groupe de travail: Ursula AckermannLiebrich, Bâle; Jacques de Haller, Genève; Beat Seiler, Zurich; Dominique Sprumont, Neuchâtel; Gianfranco Domenighetti, Lugano; Bernhard Wegmüller, Berne.

4 Au delà des indicateurs socio-économiques l'on devrait aussi considérer des indicateurs cliniques pour lesquels le «gold standard» de référence ne laisse aucune incertitude (la valeur minimale est toujours la meilleure), tels que le taux d'infections hospitalières, la mortalité opératoire, ou bien des mortalités issues de la «mortality amenable to medical intervention»: Nolte E, McKee M. Measuring the health of nations: analysis of mortality amenable to health care. Br Med J. 2003; 327:1129-32. 